\title{
Decoupled evolution of floral traits and climatic preferences in a clade of Neotropical Gesneriaceae
}

Martha Liliana Serrano-Serrano ${ }^{1,2}$, Mathieu Perret ${ }^{3}$, Maïté Guignard $^{1}$, Alain Chautems ${ }^{3}$, Daniele Silvestro ${ }^{1,2,4}$ and Nicolas Salamin ${ }^{1,2^{*}}$

\begin{abstract}
Background: Major factors influencing the phenotypic diversity of a lineage can be recognized by characterizing the extent and mode of trait evolution between related species. Here, we compared the evolutionary dynamics of traits associated with floral morphology and climatic preferences in a clade composed of the genera Codonanthopsis, Codonanthe and Nematanthus (Gesneriaceae). To test the mode and specific components that lead to phenotypic diversity in this group, we performed a Bayesian phylogenetic analysis of combined nuclear and plastid DNA sequences and modeled the evolution of quantitative traits related to flower shape and size and to climatic preferences. We propose an alternative approach to display graphically the complex dynamics of trait evolution along a phylogenetic tree using a wide range of evolutionary scenarios.
\end{abstract}

Results: Our results demonstrated heterogeneous trait evolution. Floral shapes displaced into separate regimes selected by the different pollinator types (hummingbirds versus insects), while floral size underwent a clade-specific evolution. Rates of evolution were higher for the clade that is hummingbird pollinated and experienced flower resupination, compared with species pollinated by bees, suggesting a relevant role of plant-pollinator interactions in lowland rainforest. The evolution of temperature preferences is best explained by a model with distinct selective regimes between the Brazilian Atlantic Forest and the other biomes, whereas differentiation along the precipitation axis was characterized by higher rates, compared with temperature, and no regime or clade-specific patterns.

Conclusions: Our study shows different selective regimes and clade-specific patterns in the evolution of morphological and climatic components during the diversification of Neotropical species. Our new graphical visualization tool allows the representation of trait trajectories under parameter-rich models, thus contributing to a better understanding of complex evolutionary dynamics.

Keywords: Brazilian Atlantic forest, Hummingbird pollination, Traitgram, Resupination, Pollination syndrome, Trait evolution, Comparative methods

\section{Background}

Throughout the evolutionary process, lineages may experience divergent modifications of their phenotype and genome that culminate with the establishment of separate species. Modeling the evolution of species traits can help to elucidate the likely sequence of diversification events that lead to phenotypically diverse groups of

\footnotetext{
* Correspondence: nicolas.salamin@unil.ch

'Department of Ecology and Evolution, University of Lausanne, 1015 Lausanne, Switzerland

${ }^{2}$ Swiss Institute of Bioinformatics, Quartier Sorge, 1015 Lausanne, Switzerland Full list of author information is available at the end of the article
}

species [1]. Traits related to different niche axes are expected to follow different evolutionary trajectories that may reflect different selection pressures, genetic constraints or stages of diversification determining the order in which the different ecological axes are partitioned during species divergence [2]. For example, patterns of trait divergence during the diversification of live oaks (Ceanothus) in California suggested that traits related to local scale coexistence show an early divergence in the group, while traits related to large scale habitat display a later or throughout differentiation [3]. Although 
theoretical work supports similar scenarios [1] empirical support for this model in other plant groups and across different types of traits still needs to be evaluated.

Hypotheses about the ordering of trait divergence during the evolution of a lineage can be complemented by investigating the mode and tempo of trait diversification within lineages [4]. For instance, initially rapid morphological evolution followed by relative stasis [5] could be the result of new ecological opportunities accompanied by density-dependent slowdowns in species diversification $[6,7]$. To explore this process, trait evolution can be reconstructed along the branches of phylogenetic trees to detect heterogeneity in evolutionary rates through time, across lineages or in relation to discrete characters [8-10]. Furthermore, Ornstein-Uhlenbeck (OU) models can be used to describe bounded phenotypic evolution, where single or multiple selective regimes pull phenotypes towards optimum values [11]. In plants, these models have helped to understand the evolutionary dynamics of flower morphology [12] and climatic niche [13]. Multiple studies have identified heterogeneous rates of evolution across climate dimensions in specific clades [14] and, at a larger scale, rates of niche evolution within major groups of angiosperms that are dependent on the type of growth form [15]. The possibility of testing multiple models to reveal complex patterns of trait evolution during species diversification is an important advantage to understand the dynamics of trait evolution and differential evolution among traits [7, 16, 17]. However, the fit between the current models and the real evolutionary processes is widely discussed [5, 18], and the power for selecting models depends on the number of taxa, the shape of the phylogeny, and the presence of measurements errors [19].

In this study, we investigate the evolutionary history of floral morphology and climatic preferences in a clade of epiphytic plants belonging to the genera Codonanthopsis, Codonanthe, and Nematanthus (hereafter referred to as the CCN clade) of the Gesneriaceae family. This group provides an excellent opportunity to compare patterns of evolutionary diversification between these niche axes. CCN clade exhibits a remarkable floral diversity in shape, size and orientation reflecting potential adaptation to different pollinators including bees and various hummingbirds [20-25]. Furthermore, CCN clade is widely distributed throughout most Neotropical rainforest but present the highest species richness and level of range overlap in the Brazilian Atlantic Forest (BAF) $[26,27]$. Understanding how these morphological or climatic axes of niche differentiation have evolved in this plant group could shed light on the way speciation processes are building Neotropical biodiversity. First, we test if traits related to flower shape and size better fits a pollinator shift model involving transitions between adaptive peaks defined by pollinator morphology and behavior [12], or if flowers have diversified regardless of the pollinator type. Second, we determine if the evolution of climatic preferences is best explained by a model with distinct ecological optima [13] or a model with more labile evolution of climatic preferences among closely related species [14]. To address these questions we first infer phylogenetic relationships among the species using multi-gene DNA sequences. We quantify the floral morphology and climatic space occupied by the group and, examine the tempo and mode of evolution of different traits in the $\mathrm{CCN}$ group using current models of trait evolution. We finally develop a new approach to visualize the estimated trait evolution by proposing an alternative way to incorporate information from complex models. Our results suggest that phenotypic evolution of this group is described by a variety of processes with different mode, time and lineage-specific effects. A new visualization of complex models of trait evolution further allows a better understanding of the particular processes at play in this group of Neotropical plants.

\section{Methods}

\section{Phylogenetic analyses}

Taxonomic sampling included 46 out of the 52 species in the group, as well as 13 outgroup species. Six molecular makers, two nuclear (ITS and ncpGS) and four plastid regions $(a t p B-r b c L$ spacer, rpl16 intron, rps16 intron, $\operatorname{trnL}$-trnF spacer) were sequenced and aligned for a final DNA matrix of $4.484 \mathrm{bp}$. We reconstructed phylogenetic relationships and relative divergence times using MrBayes and BEAST [28, 29]. Best fitting nucleotide substitution models were estimated with the phymltest function in the R package ape [30]. Log-normal uncorrelated relaxed clock and the Yule speciation priors were set for the analyses. We used a maximum clade credibility (MCC) tree and a sample of high posterior probability trees from the BEAST results for later analyses. Finally, we examined the evolution of three binary traits (geographic distribution, pollination syndromes, and floral orientation) by reconstructing their ancestral states in the R package corHMM [31]. Detailed description of the molecular dataset, phylogenetic reconstructions, and ancestral state estimation are provided in the Additional file 1: Appendix S1.

\section{Morphometric data}

Thirteen quantitative traits representing different aspects of the floral shape and size (Additional file 1: Figure S1) were measured for 38 species out of the 46 included in the phylogenetic analysis (Additional file 1: Table S3). Measurements were obtained from 2 to 15 flowers 
(average $=5$ ) collected from wild individuals or cultivated plants at the Botanical Garden of Geneva (Switzerland). Collection permits were granted by the CNPq in Brazil (CMC 038/03) and the ANAM in Panama (SC/P-43-10). Floral material was not available for Codonanthopsis dissimulata, five species of Codonanthe and two Nematanthus (C. crassifolia, C. calcarata, C. gibbosa, C. erubescens, C. luteola, N. kautskyi, and $N$. lanceolatus). However, original descriptions of the species and photographic material available at Mauro Peixoto website (www.brazilplants.com) and at the Gesneriaceae Image Library (http://gesneriads.ua.edu/image-library/) indicates that the missing species do not represent exceptional morpho-types of the group. Thus, we can postulate that our quantitative measurements are representative of the morphological diversity of each clade and that we do not miss important variation because of the species lacking morphological data. Species positions in morphological space was quantified with a principal component analysis (PCA) using the R package Ade4 [32] based on the covariance matrix of mean values for each species. We used nontransformed data, but log-transformed PCA patterns were also examined and led to a very similar morphospace (see Additional file 1: Figure S5).

\section{Species climatic preferences}

Climatic parameters for each species of the $\mathrm{CCN}$ clade were estimated from occurrence data and layers for climatic data. Locality descriptions were derived from the labels on specimens examined in more than 50 herbaria (Additional file 1: Table S4). Georeference coordinates were generated for all localities that could be attributed to a precise geographic entity. We completed the dataset for species occurring outside Brazil with additional georeferenced specimens retrieved from GBIF (data.gbif.org, 2012-02-06). Occurrence data for C. corniculata, C. elegans and $N$. serpens were not included in the analysis due to limited number of herbarium material and uncertainty on their native distribution. A total of 2,240 occurrence points remained after manual checking and removal of duplicated points with a median of 15 occurrences per species. Climatic data (elevation and 19 bioclimatic variables) were extracted directly from Bioclim environmental layers [33] on a 30 arc-second resolution grid $\left(\sim 1 \mathrm{~km}^{2}\right.$ at the equator). Occurrence data and their associated climatic information extracted from the Bioclim layers can be found at http://www2.unil.ch/phylo/ files/serranoetal15_bioclim.xls. We used these climatic parameters to represent the species distribution along climatic gradients. We denoted this climatic space as the species preferences, although these preferences may be limited by interactions with other species, historical factors or dispersal limitation [34]. To explore the relative position of each species in the climatic space of the $\mathrm{CCN}$ clade, we performed a PCA using outlying mean index ordination (OMI) [35], which assigns a mean position of each species on the climatic space, as implemented in Ade4 package in R [32]. Values for each of the 19 Bioclim variables, plus altitude, were used for the ordination.

\section{Models of continuous trait evolution}

We examined the patterns of trait evolution by using the MCC tree and multiple models, which span from a single Brownian motion rate of evolution (BM with single $\sigma^{2}$ ), BM with variable rate through time (decreasing or increasing $\sigma^{2}$ ) including early burst (EB) to OrnsteinUhlenbeck models. All these models were fitted using the fitContinuous function in geiger R package [36]. The Ornstein-Uhlenbeck models were fitted using either a single selective regime (one single $\sigma^{2}$, selection strength $\alpha$ and optimum parameter $\theta$ ) or multi-regime processes. For the latter case, we tested four different OU models using the OUwie function in the OUwie package [11] (Additional file 1: Table S6 for acronyms), each with two different regime categories: pollination syndromes and geographic distribution. These categories were treated as binary characters, following the reconstructions described in Additional file 1: Appendix S1. Delta-AIC $(\triangle \mathrm{AIC})$ and Akaike weights $(\omega)$ were calculated for model comparisons. Furthermore, Blomberg's K [37] was estimated as a measure of phylogenetic signal using the $\mathrm{R}$ package phytools [38]. The outgroup species were pruned from the trees in all the morphology and climatic preferences analyses.

In addition to the models of trait evolution, a multirate $\mathrm{BM}$ model was tested using the $\mathrm{R}$ package geiger to identify rate changes among lineages [36]. This flexible method aims to identify changes on rates of continuous trait evolution among lineages. The analyses were performed on 100 phylogenetic trees randomly sampled from the BEAST posterior distribution and the MCMC was run for 100,000 generations, sampling every 100 generations and excluding the first $25 \%$ for burn-in. Each run provides posterior distributions of branch-wise rate estimates and probabilities of rate shifts. We compared the fit of the multi-rates model against the fit of alternative BM and OU models by comparing their AIC value. The multi-rates $\mathrm{BM}$ analysis used reversible jump MCMC (rjMCMC) and we estimated the AIC values for each model in two ways. First, we directly took the best likelihood value sampled over the riMCMC samples and calculate the corresponding AIC value based on the number of parameters of the model. Second, we mapped the set of branches at each rate category with the make.era.map function, and used the non-censored approach implemented in the brownie.lite function 
(phytools R package) [38] to produce the maximum likelihood estimate and calculate the AIC values. A bias towards parameter-rich models can occur during the model selection process if measurement error is not considered [19]. We performed additional model comparisons to test for such effects by estimating the amount of measurement error present in our dataset. Such estimation is not possible for multi-rates BM model but we used the values estimated under single BM model estimation for these cases.

\section{Visualization of continuous trait evolution}

The parameters estimated under the best fitting model of trait evolution describe the process numerically, but a graphical visualization of trait evolutionary trajectories remains difficult to picture. Recent graphical methods (often referred to as "traitgrams") help to visualize phenotypic evolution by plotting a phylogenetic tree against trait values [39]. However, such methods are limited to the BM model, partly due to the difficulty of inferring ancestral states under more complex evolutionary models. Here, we implemented an alternative approach to display the dynamics of trait evolution along a phylogenetic tree. We achieved this by forward-time simulations under complex models of trait evolution and analytical interpolation between ancestral states. The purpose of these reconstructions is to provide a graphical representation of the expected continuous evolution of phenotypes given a phylogeny and a set of parameters describing the evolutionary process. For each trait, we recorded the topological placement and magnitude of parameter changes across the tree, e.g. rates, selection strength and optima, depending on the model (Table 1). We then simulated 100 realizations of trait evolution under the optimized model and parameter values along the BEAST MCC tree (see Appendix S2 for more details). All forward simulations started by sampling a random number from a normal distribution with parameters estimated from a posterior distribution of root states obtained with the fitContinuousMCMC function in geiger [36]. Other parameters $\left(\sigma^{2}, \alpha, \theta\right)$ were taken from the best fitting model.

We used the simulated trait values at the internal nodes and at the tips to plot traitgrams displaying the reconstructed trait evolution. In their standard implementation traitgrams connects the trait values between nodes by a straight line to draw the edges of a tree $[39,40]$, consistently with the expected anagenetic trait evolution under a constant rate BM model. However, under more complex models such as OU processes, the expected trait value $x(t)$ at a time $t$ between two nodes of age $t_{i}<t<t_{j}$ and trait values $x\left(t_{i}\right)$ and $x\left(t_{j}\right)$ respectively, deviates from a straight line. This deviation occurs because the evolutionary trajectory of the trait under an OU model depends not only on the evolutionary rate, but also on the strength of
Table 1 Results of model fitting for the morphological and climate PC axes

\begin{tabular}{lccl}
\hline Models & \multicolumn{1}{l}{ AlCC } & \multicolumn{1}{l}{$\Delta$ AIC } & $\omega$ \\
\hline Floral size - Morphology PC1 & & & \\
Brownian Motion & 190.9718 & 38.7133 & 0.0000 \\
Ornstein-Uhlenbeck & 187.7321 & 35.4736 & 0.0000 \\
OU alternative ${ }^{\mathrm{a}}$ & 172.9199 & 20.6614 & 0.0000 \\
Early Burst (DC) & 193.3461 & 41.0876 & 0.0000 \\
Early burst (AC) & 187.7321 & 35.4736 & 0.0000 \\
Multiple rates & 152.2585 & 0.0000 & 1.0000 \\
Floral shape - Morphology PC2 & & & \\
Brownian Motion & 104.3669 & 24.8268 & 0.0000 \\
Ornstein-Uhlenbeck & 106.6641 & 27.1240 & 0.0000 \\
OU alternative & & & \\
Early Burst (DC) & 79.5401 & 0.0000 & 0.9996 \\
Early burst (AC) & 106.7412 & 27.2011 & 0.0000 \\
Multiple rates & 106.6641 & 27.1240 & 0.0000 \\
& 95.2293 & 15.6892 & 0.0004
\end{tabular}

Mean and seasonality in temperature - Climate PC1

$\begin{array}{lrrr}\text { Brownian Motion } & 134.5776 & 10.1545 & 0.0059 \\ \text { Ornstein-Uhlenbeck } & 135.6864 & 11.2633 & 0.0034 \\ \text { OU alternative }{ }^{\mathrm{a}} & 124.4231 & 0.0000 & 0.9465 \\ \text { Early Burst (DC) } & 136.9015 & 12.4784 & 0.0018 \\ \text { Early burst (AC) } & 136.5797 & 12.1566 & 0.0022 \\ \text { Multiple rates } & 130.7428 & 6.3197 & 0.0402 \\ \text { Precipitation seasonality - Climate PC2 } & & \\ \text { Brownian Motion } & 115.0848 & 19.9517 & 0.0000 \\ \text { Ornstein-Uhlenbeck } & 100.5829 & 5.4498 & 0.0548 \\ \text { OU alternative } & 100.5768 & 5.4437 & 0.0549 \\ \text { Early Burst (DC) } & 117.4086 & 22.2755 & 0.0000 \\ \text { Early burst (AC) } & 100.5829 & 5.4498 & 0.0548 \\ \text { Multiple rates } & 95.1331 & 0.0000 & 0.8355\end{array}$

${ }^{\mathrm{a}} \mathrm{OU}$ alternative corresponds to Ornstein-Uhlenbeck models with different numbers of parameters. Here, only the model with the best AIC value is reported, see the full set of OU models in summarized in the Additional file 1: Table S6. (AICC = corrected Akaike Information Criterion values, $\triangle \mathrm{AIC}$ Delta AICc and $\omega=$ Akaike weights)

selection and the relative distance from the optimum [41]. The expected anagenetic evolution of a trait under an $\mathrm{OU}$ model is described by the Ornstein-Uhlenbeck bridge (personal communication) [42] and can be obtained for any time $t$ as:

$$
\begin{aligned}
x(t)=\theta & +\frac{\left(x\left(t_{i}\right)-\theta\right) \sinh \left(\alpha\left(t_{j}-t\right)\right)}{\sinh \left(\alpha\left(t_{j}-t_{i}\right)\right)} \\
& +\frac{\left(x\left(t_{i}\right)-\theta\right) \sinh \left(\alpha\left(t-t_{i}\right)\right)}{\sinh \left(\alpha\left(t_{j}-t_{i}\right)\right)}, \text { for } t_{i}<t<t_{j}
\end{aligned}
$$

where $\theta$ is the optimum and $\alpha$ the strength of selection. The parameters $\theta$ and $\alpha$ can vary across the edges of a tree if the model includes different selective regimes. 
We generated multiple realizations of the trait evolution under the different BM and OU models and parameter settings estimated for the four traits. These were combined to plot the $95 \% \mathrm{CI}$ of the trait ranges through time (i.e., the minimum and maximum trait values across clades at any time $t$ ). These plots thus provide a graphical representation of how the range of potential trait values are expected to change through time, given a fixed tree topology and a complex model of trait evolution. Single realizations of the simulated process, resembling the conventional traitgrams, facilitate the understanding of regime- or clade-specific patterns. The method is implemented in an $\mathrm{R}$ script available at http://www2.unil.ch/ phylo/files/software/plot_traitgram_serranoetal15.R.

\section{Results}

\section{Phylogenetic analyses}

The tree topologies reconstructed from MrBayes and BEAST were congruent and generally highly supported (see Additional file 1: Figures S2 and S6). The BEAST analysis (Fig. 1, see Additional file 1: Figure S2 for tree with outgroups) indicated that the initial divergence in the $\mathrm{CCN}$ lineage occurred between the Codonanthopsis clade and the remaining taxa that are all endemic to the BAF. The Codonanthopsis clade is widely distributed in Central America, the Caribbean, the Andes and the Amazonian basin, but does not occur in the BAF except for the widespread C. uleana, which extends its range into the northern part of this biome. The BAF clade is composed of the two sister clades corresponding to the Codonanthe s.s. (seven species) and Nematanthus (27 species). Within Nematanthus, the sister species N. australis and $N$. wettsteinii first diverged from the remaining Nematanthus, which includes two sister clades identified as Nematanthus $A$ (17 species) and Nematanthus $B$ (7 species). High posterior probabilities supported these clades (1.0), while ambiguous placements were observed for C. mattos-silvae, N. kaustkyi and $N$. hirtellus species. Ancestral reconstructions for geography, pollination syndrome and floral orientation using the preferred model (ER) showed few state changes, mainly involving complete clade transitions (Additional file 1: Figure S3).

\section{Floral morphology and climatic preferences space}

The first and second axes of the PCA on the floral traits explained $85.1 \%$ of the variance (PC1 $70.7 \%$; $\mathrm{PC} 2$ 14.4 \%, Additional file 1: Figure S4a and Table S5). PC1 mainly reflected variation in flower size with loadings of same sign and approximately equal value for all measurements. Variation in flower size (PC1) was particularly extensive in the Nematanthus-A clade (Additional file 1: Figure S4a). PC2 had a positive loading for stamen, pistil and tube lengths, vertical diameter of corolla tube, and a negative loading for the diameters of limb and corolla opening and restriction before nectary chamber. PC2 therefore mainly represented variation in flower shape, with a positive value indicating tubular and narrowly opened corolla, while a negative value indicates more campanulate corolla with inserted stamen and broad limb and opening. All species with positive value of PC2 belong to the genus Nematanthus. Their flowers are pigmented in red, orange or yellow and match well the definition of the syndrome of hummingbird pollination (Fenster et al. 2004), as confirmed by field studies for 10 species (see Additional file 1: Table S2). Species with negative PC2 values belong to the Codonanthe, Codonanthopsis and to a specific subclade within Nematanthus-A. Their flowers have several features traditionally associated with bee pollination such as the creamy corolla, brownish dots inside the tube (nectary guide), inserted stamen and developed inferior lobes forming a landing platform for insects. However, to our knowledge, no field studies have confirmed bee pollination for these species.

The first two PCs for climatic variables accounted for $69.1 \%$ of the variance (PC1 $54.30 \%$, PC2 $14.84 \%$, Additional file 1: Figure S4b). PC1 reflected mainly the variation in temperature (Bio9, Bio11, Bio4 and Bio7) with positive values indicating warmer mean temperatures with low seasonal variation and negative values showing strong variability in temperature through the year. PC2 was mainly correlated with precipitation (Bio15 and Bio14). Positive PC2 values indicated high seasonal variability in precipitation regimes, while negative values showed high precipitation on the wettest month and low seasonality (see Additional file 1: Figure S4b for species climatic values). Climatic space indicated a clear separation between the Codonanthopsis species and the BAF lineages (Codonanthe plus Nematanthus clades), in agreement with their distinct geographical distribution.

\section{Models of continuous trait evolution}

The models of trait evolution indicated that distinct evolutionary processes have influenced trait divergence in the CCN group. Individual axes of floral morphology (size and shape) and climatic preferences (temperature and precipitation) variation seemed to have evolved independently. The incorporation of measurement error in our model comparisons did not lead to any bias towards parameter-rich models and the four phenotypic axes present no changes in the preferred models when accounting for it (Additional file 1: Table S7).

The evolution of floral size (PC1) was best described by the multi-rates $\mathrm{BM}$ model with branch-specific rates (Table 1 ). The $\triangle \mathrm{AIC}$ is very large suggesting that the alternative models poorly represent the evolution of the floral size. The posterior probabilities for the rates of 


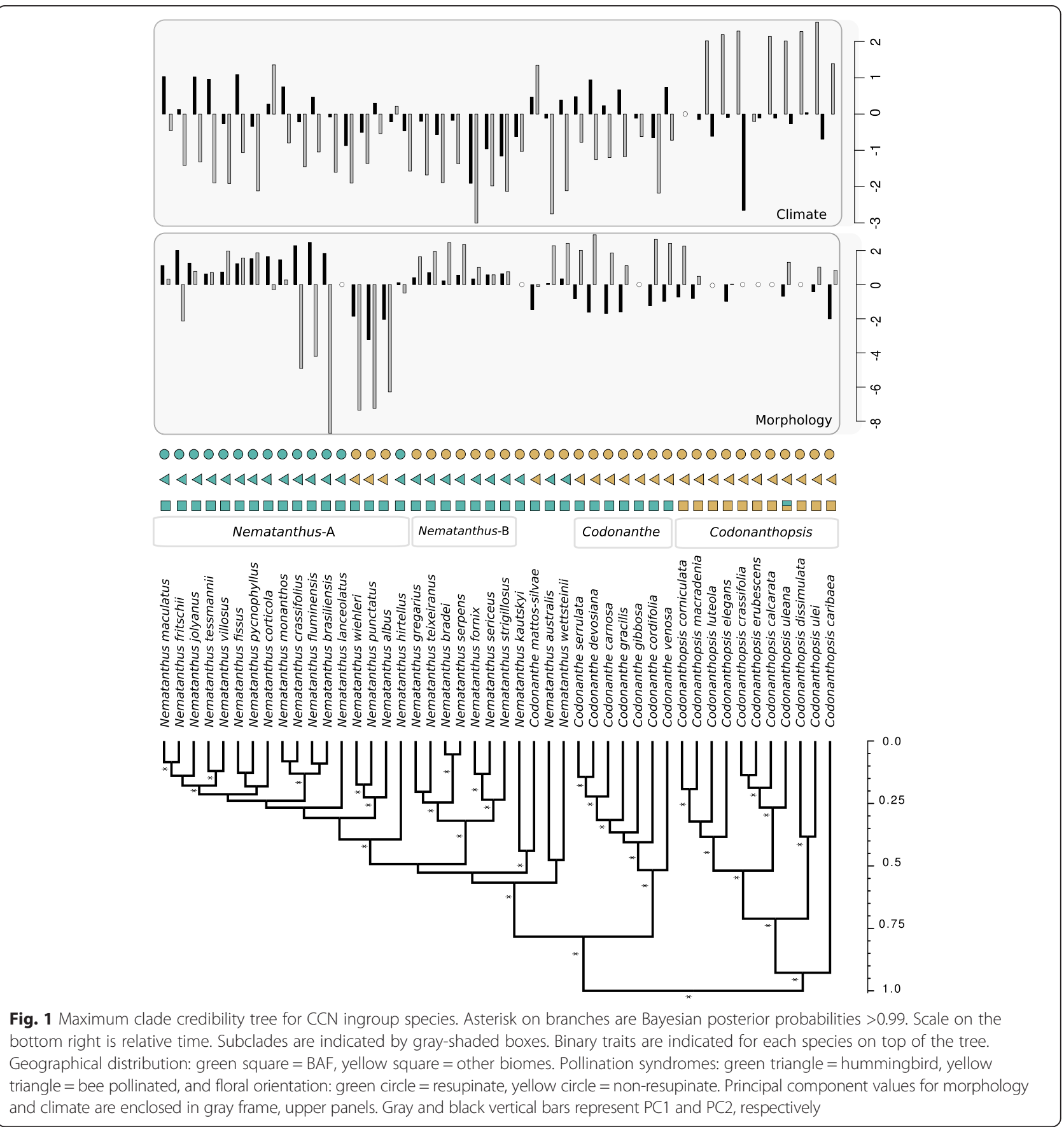

evolution across the phylogeny supported one rate shift in this trait (Fig. 3), which is associated with the origin of the Nematanthus-A clade (shift probability >0.625). This shift involved a strong rate increase and represents a deviation from a constant BM process, which is consistent with the observed low values of Blomberg's $\mathrm{K}$ statistic for PC1 (mean of 0.496 with $25 \%$ and $75 \%$ quantiles of 0.463 and 0.546 , respectively). According to the parameter estimated by the best fitting model, the visualization of floral size evolution shows narrow trait ranges in the early stages of the diversification of the $\mathrm{CCN}$ group, followed by a large increase in trait ranges due to the single clade shift (Fig. 2a). From the simulated trait trajectories, and because the best model was based on $\mathrm{BM}$, the increase in trait range can be symmetric (positive or negative axis). However, the empirical data suggested that only deviation towards bigger floral sizes occurred (negative loadings). The evolution of the floral shape (PC2) was best explained by an OUMVA model with two regimes, which are defined by the pollination 


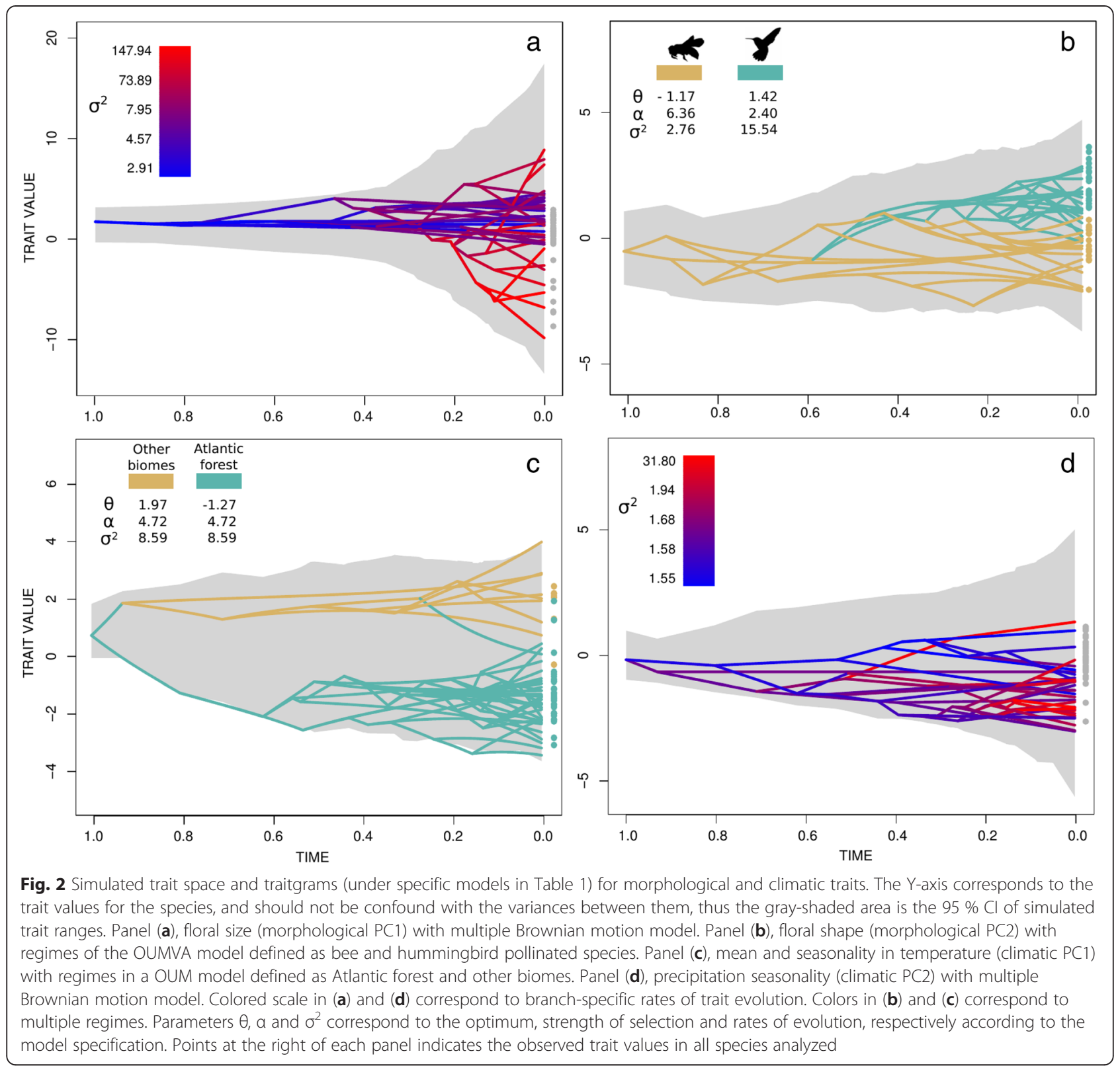

types. Each regime had different rates of evolution $\left(\sigma^{2}\right)$, optima $(\theta)$ and strength of selection $(\alpha)$. Bee-pollinated species (negative loadings in the PC2) evolved in a constrained way, with a narrower dispersion from the optimum value, whereas hummingbird-pollinated species (positive loadings in PC2) explored a wider trait space during their evolution (Fig. 2b). Phylogenetic reconstruction indicated a single origin of hummingbird syndrome at the root of the Nematanthus clade and more recent reversal to bee syndrome in a clade of three species ( $N$. albus, $N$. punctatus, and $N$. wiehleri, Additional file 1: Figure S3). Floral shape constraint agrees with the high Blomberg's $\mathrm{K}$ values obtained for the morphological PC2 (mean value of 1.323 with $25 \%$ and $75 \%$ quantiles of 1.122 and 1.547, respectively).

The evolution of climatic preferences also showed different dynamics among its components. The best model for temperature (climate PC1) was OUM, with different optima between the two geographic distributions (i.e., Central, northern South America and Amazonian basin versus Brazilian Atlantic forest), but equal rates of evolution and selection coefficient per regime. The trait space for temperature displayed a strongly bounded evolution with a slow rate of change (Fig. 2c). The extent of change in positive and negative loadings appeared to be 
symmetrical. Estimates of Blomberg's $\mathrm{K}$ statistic for climatic PC1 indicated a mean value of 0.477 with $25 \%$ and $75 \%$ quantiles of 0.376 and 0.541 , respectively. Multi-rates BM model was the best fitted for precipitation seasonality (climatic PC2), but posterior evidence for a rate shift was weak. Only a minor increase in rates of climatic differentiation between the sister species $C$. erubescens and C. crassifolia was detected (see Fig. 4). The evolution of trait space for the climatic PC2 appeared as a constant increase of phenotypic space over time (Fig. 2d). This was consistent with the results in Fig. 4, showing that large shifts in the trait values are rare. Estimates of Blomberg's K values for climatic PC2 ranged from 0.357 and 0.459 (25\% and $75 \%$ quantiles respectively) with a mean value of 0.408 .

\section{Discussion}

Testing the order and extent of trait divergence during the evolution of a clade helps to understand the relative importance of separate morphological and climatic trajectories, as well as the possible drivers of species diversification. We combine a phylogenetic analysis and multiple models of trait evolution with simulations under the selected models, in order to comprehensively understand these different trajectories of trait evolution in the $\mathrm{CCN}$ group. Our results suggest that phenotypic evolution of this group is described by a variety of processes with different mode, time and lineage-specific effects. A new visualization of complex models of trait evolution further allow a better understanding of the particular processes at play in this group of Neotropical plants.

\section{Floral evolution dynamics}

The inference of evolutionary models and estimation of plausible trait ranges for floral morphology revealed contrasting patterns during the evolutionary history of the $\mathrm{CCN}$ group. Floral size, represented by morphological PC1, has evolved in a complex fashion. The estimated trait range through time showed an initial period of narrow divergence, followed by a marked increase in trait ranges associated with the accelerated evolution of flower size within the clade Nematanthus-A (Fig. 2a). We did not detect evidence of a slow down in the rate of evolution of the $\mathrm{PC} 1$, showing that divergence in floral size continues throughout the diversification of the Nematanthus- $A$ lineage during the Miocene (24 Mya, $95 \%$ HPD 33.45 - 9.30 Mya [27]). This result contrasts with the classical model of adaptive radiation, where morphological evolution is initially rapid and then slows through time [5]. Our analyses were not aimed at investigating whether the $\mathrm{CCN}$ clade is a case of adaptive radiation, however the lack of a slow down in the evolutionary trajectory of floral size suggest that morphological space is not yet filled.

The increase in rates of evolution of floral size detected at the base of the Nematanthus-A clade coincides with the evolution of floral resupination (see the placement of the rate shift in Fig. 3, and the most probable transition to resupinate flowers occurring in almost all species of the clade except for $N$. albus, $N$. wiehleri and N. punctatus in Fig. S3). A direct consequence of the evolution of flower resupination is a change of pollen placement on the body of the pollinator. In resupinate species belonging to the Nematanthus-A clade, pollen is primarily transported on different parts of the ventral side of hummingbirds $[20,23]$. In contrast, nonresupinate Nematanthus place most of their pollen on the top of the bill [22]. Therefore, flower resupination and the associated shift of pollen deposition could have stimulated the diversification of floral size in Nematanthus-A clade by creating new opportunities for species coexistence while sharing pollinators as was shown in another community of hummingbird-pollinated plants [43]. Extending this analysis to other Gesneriaceae clades such as Glossoloma and Crantzia that independently evolved resupinate flowers pollinated by hummingbirds, would provide a mean to further test the positive effect of resupination on flower diversification [44, 45]. An additional feature of the Nematanthus-A clade is its contrasting altitudinal distribution compared with Nematanthus-B (median values of $524 \mathrm{~m}$ and $957 \mathrm{~m}$, respectively). In the BAF, these two altitudinal levels have contrasting hummingbird assemblages, with lowland communities presenting a greater richness of hummingbirds and a corresponding higher heterogeneity in bill size (short-billed trochilines and long-billed hermits) compared with the highland sites [21, 22]. Indeed, Nematanthus-A species in lowland rainforest are visited by both Trochilinae (non-hermit) and long-billed hermit hummingbirds (Phaethornis and Ramphodon), whereas species from Nematanthus-B mainly rely on Trochilinae hummingbirds for pollination (see Additional file 1: Table S2). We suggest that the interaction of Nematanthus- $A$ species with broader range of hummingbird types and bill lengths in the lowland forest could also have provided more opportunities for flower size diversification in this clade.

Contrary to flower size, the evolution of the morphological PC2, floral shape, was preferentially supported by OU models that contained differential strength of selection, optima and rate parameters between hummingbird and bee pollination syndromes. The evolution of floral shape, from funnel-shaped corolla with expanded lobes in Codonanthopsis and Codonanthe clades to narrow-mouth and pouched corollas in most Nematanthus species could have been differentially constrained and maintained by 


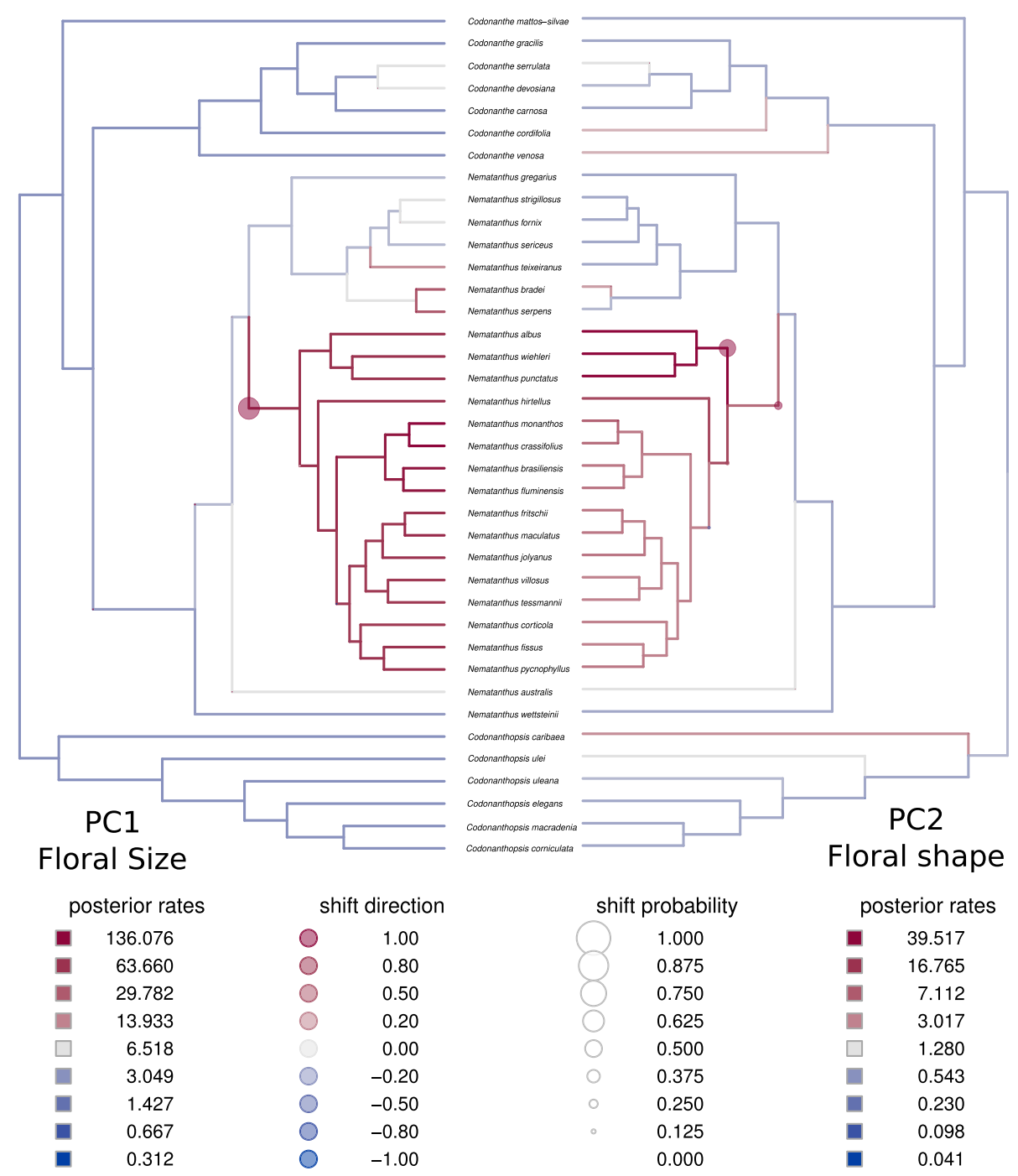

Fig. 3 Posterior comparisons of rate of morphological trait evolution in the CCN group. Hue and size of circles at branches denote posterior support for a rate shift at the indicated branch. Larger and redder circles suggest higher posterior support for an upturn in evolutionary rate (see Eastman et al. 2011). Branches in the phylogeny are colored such that rates not deviant from the median are shaded gray; rates below (or above) the median are shaded blue (or red). Rates corresponding to each hue are indicated in the legend, as well as shift probabilities and directions

functional groups of pollinators in agreement with the pollination syndrome concept [46]. Hummingbirdpollinated species showed a lower strength of selection and higher rates of trait change than species with a bee pollination syndrome (parameters in Fig. 2b), suggesting that hummingbirds might interact with a wider range of flower shapes than bees. The visualization of the floral shape trait space in this group provides a convenient tool that helps to better understand the dynamics of the $\mathrm{OU}$ process and the evolution of the independent optima.

Overall, our results suggest that the evolutionary trajectory of floral morphology in the $\mathrm{CCN}$ group may be constrained in shape (PC2), with possible evolutionary transitions from one functional group of pollinators to another following the pollinator shift model [12]. The transitions between these two phenotypic clusters may reflect selection to improve the interaction with better pollinators [46], and/or to avoid less efficient floralpollinator associations $[47,48]$. In comparison, variation in floral size (PC1) could be more related to character displacement and the establishment of mechanical isolation between co-occurring species sharing a same functional group of pollinators $[49,50]$. This model of flower evolution based on competition for hummingbird pollination has been shown to generate phenotypic overdispersion within communities of Andean Solanaceae [51]. Testing this prediction in the $\mathrm{CCN}$ clade would require to further investigate whether co-occurring species are more different in flower size than expected due to chance across different sites in the BAF. 


\section{Climatic evolution dynamics}

The evolution of climatic preferences, represented in PC1 by temperature, is best explained by an OU model (Table 1). The different optima in the OUM model indicate the differentiation between BAF and other rainforests in the Neotropical region. However, models accounting for different rates of evolution and/or strength of selection are not preferred. This result potentially indicates that species preferences in temperature evolve at a similar pace, and that the strength of selection is comparable between groups inhabiting different regions. This pattern could reflect specific regional variation and dispersal limitation among the $\mathrm{CCN}$ lineages. The graphical representation of the evolution of the temperature preference showed an early differentiation between the selective regimes with rare subsequent transitions from one biome to the other (Fig. 2c). In contrast, the multi-rate BM model best explained the precipitation preferences, which are represented by $\mathrm{PC} 2$. The estimated rates for this component were higher than the overall rates in PC1 (see parameters in Fig. 2c and branch-specific estimations in Fig. 4), suggesting that species preferences for precipitation seasonality might change more rapidly than temperature preferences which are mainly biome specific.

Several evolutionary studies have reported pronounced ecological niche differentiation, concentrated in particular lineages $[13,14,52]$ or associated with distinct species traits $[53,54]$, which suggest an important role of climatic changes during speciation. Our results for climatic differentiation suggest an early biome separation of the CCN clades (BAF and the rest of the Neotropics), followed by a divergence along different local conditions of precipitation seasonality. Although the role of this climatic component in speciation would need to be further investigated, this result is consistent with previous studies showing that floristic turnover in the Atlantic forests is largely correlated with distance from the ocean and rainfall distribution patterns $[55,56]$ and that allopatric speciation could have been particularly frequent along this climatic gradient $[57,58]$.

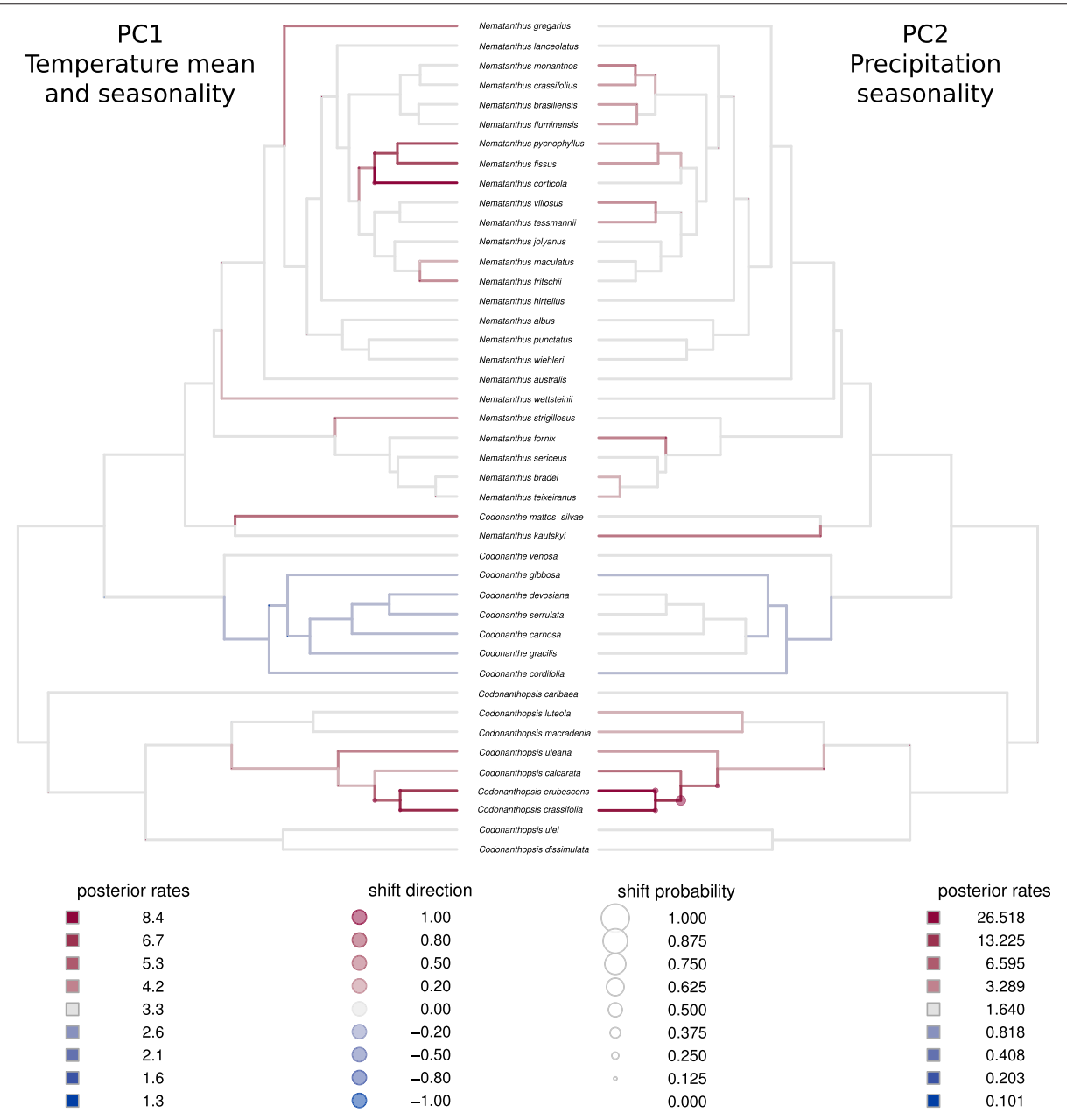

Fig. 4 Posterior comparisons of rate of climatic preferences evolution in the CCN group. See caption of Fig. 3 


\section{Conclusions}

Our investigation of the mode and tempo of trait evolution in the CCN clade provided evidence for a contrasting relevance of morphological and ecological divergences during species diversification. Two traits flower shape and temperature preferences - were segregated into adaptive zones associated with different functional group of pollinators or biogeographic regions. First, floral shape evolution was constrained reflecting the selection to different functional groups of pollinators (i.e. hummingbirds vs insects). Second, divergence in temperature was linked with the colonization of the BAF biome at an early stage of the evolution of the CCN group. On the contrary, two other trait components flower size and precipitation preferences - evolved at a higher rate, with no recent slowdown. Changes in floral size occurred mainly in a specific subclade including species with resupinate flowers and lowland distribution, whereas evolutionary changes of precipitation seasonality likely took place tree-wide and throughout the entire $\mathrm{CCN}$ diversification. The contrasting patterns between the constrained evolution of floral shape (i.e. pollination syndrome) and the diversification of precipitation seasonality across time agree with the habitat first rule model proposed by Ackerly et al. (2006) suggesting early divergence of traits that allow species to co-occur (alpha niche) and a throughout diversification of traits defining macrohabitats (beta niche). We found, however, that both flower morphology and climatic preferences diversified along different axes that are better fitted by distinct models of evolution. Our new implementation for visualization allowed us to graphically represent trait evolutionary histories, using models that go beyond the simple BM and can potentially better capture complex evolutionary dynamics. This approach represents an enhancement of current methods to plot the phenotypic space through time, with the simulation part being of potential use as a predictive tool for measuring the adequacy of alternative models. Finally, our study calls for a broader phylogenetic scale analysis to unravel the mechanisms driving such evolutionary processes and their potential effect on the remarkable species richness in the Neotropics.

\section{Additional file}

Additional file 1: Appendix S1. Phylogenetic analysis and relative divergence time estimation. Appendix S2. Visualization of continuous trait evolution. Table S1. List of species sampled, voucher information, Genbank accession numbers and binary trait information. Table S2. Pollinator, locality and source of information for Nematanthus species. Table S3. Mean values for floral traits. Table S4. Index Herbariorum acronyms and names. Table S5. PC loadings for morphological and climatic preferences. Table S6. AICc values for the complete set of OU models. Table S7. Model fit comparison correcting for measurement error (ME). Figure S1. Floral measurements. Figure S2. Phylogenetic reconstruction with outgroup species. Figure S3. Ancestral state reconstructions. Figure S4. Principal component analysis for floral traits (a) and climatic preferences (b). Figure S5. Comparison of phenotypic space and multi-rates BM model from raw and log-transformed measurements. Figure S6. Credibility tree from MrBayes with outgroup sequences included. (PDF $4115 \mathrm{~kb}$ )

\section{Competing interests}

All authors declare that they have no competing interests.

\section{Authors' contributions}

MLSS and NS conceived the study. MP, AC and MG participated significantly in the production of the data. MLSS performed lab work and analyses with the help of DS. NS and MP supervised MLSS, coordinated the project and helped to draft the manuscript. All authors have read and approved the manuscript.

\section{Authors' information}

MLSS is a PhD student in the group of NS. NS is a Professor at the University of Lausanne. NS research group focuses on a variety of organisms and questions with a particular interest on understanding the drivers of adaptation both at the levels of the genes or genomes but also through the modeling of the evolution of the phenotypes and the ecological niche of species.

\section{Acknowledgements}

We thank Régine Niba and Fadil Avdija for their contribution to the laboratory work; Pierre Mattille, Yvonne Menneret, Marina Magnette, and Matthieu Grillet for the propagation and maintenance of the Gesneriaceae collection at Geneva. Collection permits were granted by the CNPq in Brazil (CMC 038/03) and the ANAM in Panama (SC/P-43-10). We thank Mauro Peixoto for the kind supply of the photographic and plant material. We also thank Linda Dib, Anna Kostikova, Sacha Laurent, Glenn Litsios, Hervé Sauquet and Sylvain Corlay for the helpful discussions. We thank Simon Joly and an anonymous reviewer for insightful comments that greatly improved the manuscript. All analyses were run at the High-performance Computing Center (Vital-IT) from the Swiss Institute of Bioinformatics. This study was funded by the Faculty of Biology and Medicine at the University of Lausanne and the Swiss National Science foundation (grant number CRSII3_147630) DS is funded by the Wenner-Gren Foundation and Carl Tryggers stiftelse (Sweden)

\section{Author details}

${ }^{1}$ Department of Ecology and Evolution, University of Lausanne, 1015 Lausanne, Switzerland. ${ }^{2}$ Swiss Institute of Bioinformatics, Quartier Sorge, 1015 Lausanne, Switzerland. ${ }^{3}$ Conservatoire et Jardin botaniques de la Ville de Genève and Laboratory of Plant Systematics and Biodiversity, University of Geneva, Chemin de I'Impératrice, 1, 1292 Chambésy, Geneva, Switzerland. ${ }^{4}$ Department of Plant and Environmental Sciences, University of Gothenburg, Carl Skottsbergs gata 22B, 41319 Gothenburg, Sweden.

Received: 24 February 2015 Accepted: 30 October 2015

Published online: 10 November 2015

\section{References}

1. Gravilets S, Losos J. Adaptive radiation: contrasting theory with data. Science. 2009:323:732-7.

2. Schluter $D$. The ecology of adaptive radiation. Oxford: Oxford University Press; 2000.

3. Ackerly D, Schwilk D, Webb CO. Niche evolution and adaptive radiation: testing the order of trait divergence. Ecology. 2006;87:50-61.

4. Glor RE. Phylogenetic insights on adaptive radiation. Annu Rev Ecol Evol Syst. 2010;41:251-70.

5. Harmon LJ, Losos JB, Davies TJ, Gillespie RG, Gittleman JL, Jennings WB, et al. Early bursts of body size and shape evolution are rare in comparative data. Evolution. 2010;64(8):2385-96.

6. Mahler DL, Revell LJ, Glor RE, Losos JB. Ecological opportunity and the rate of morphological evolution in the diversification of greater Antillean anoles. Evolution. 2010;64:2731-45 
7. Weir JT, Mursleen S. Diversity-dependent cladogenesis and trait evolution in the adaptive radiation of the auks (Aves: Alcidae). Evolution. 2012;67:403-16.

8. O'Meara BC, Ané C, Sanderson MJ, Wainwright PC. Testing for different rates of continuous trait evolution using likelihood. Evolution. 2006;60:922-33.

9. Eastman JM, Alfaro ME, Joyce P, Hipp AL, Harmon LJ. A novel comparative method for identifying shifts in the rate of character evolution on trees. Evolution. 2011;65(12):3578-89.

10. Revell $L$, Mahler DL, Peres-neto PR, Redelings BD. A new phylogenetic method for identifying exceptional phenotypic diversification. Evolution. 2011:66(1):135-46.

11. Beaulieu JM, Jhwueng D-C, Boettiger C, O'Meara BC. Modeling stabilizing selection: expanding the ornstein-uhlenbeck model of adaptive evolution. Evolution. 2012;66:2369-83.

12. Whittall JB, Hodges SA. Pollinator shifts drive increasingly long nectar spurs in columbine flowers. Nature Letters. 2007:447:706-10.

13. Schnitzler J, Graham CH, Dormann CF, Schiffers K, Peter Linder H. Climatic niche evolution and species diversification in the Cape flora South Africa. J Biogeogr. 2012;39:2201-11.

14. Evans MEK, Smith SA, Flynn RS, Donoghue MJ. Climate, niche evolution, and diversification of the "Bird-Cage" evening primroses (Oenothera, Sections Anogra and Kleinia). Am Nat. 2009:173:225-40.

15. Smith SA, Beaulieu JM. Life history influences rates of climatic niche evolution in flowering plants. Proc R Soc Lond Ser B. 2009;276:4345-52.

16. Kozak KH, Wiens JJ. Accelerated rates of climatic-niche evolution underlie rapid species diversification. Ecol Lett. 2010;13:1378-89.

17. Hertz PE, Arima Y, Harrison A, Huey RB, Losos JB, Glor RE. Asynchronous evolution of physiology and morphology in Anolis lizards. Evolution. 2013;67:2101-13

18. Ingram $T$, Harmon $L$, Shurin JB. When should we expect early bursts of trait evolution in comparative data? predictions from an evolutionary food web model. J Evol Biol. 2012;25:1902-10.

19. Silvestro, D., A. Kostikova, G. Litsios, P.B. Pearman, and N. Salamin. Measurement errors should always be incorporated in phylogenetic comparative analysis. Methods in Ecology and Evolution. 2015; 2041-210X.

20. Franco ALM, Buzato S. Biologia floral de Nematanthus fritschii (Gesneriaceae). Rev Brasil Biol. 1992:52:661-6.

21. Sazima I, Buzato S, Sazima M. An assemblage of hummingbird-pollinated flowers in a montane forest in southeastern Brazil. Botanica Acta. 1996;109:149-60.

22. Buzato S, Sazima M, Sazima I. Hummingbird-pollinated floras at three Atlantic forest sites. Biotropica. 2000;32:824-41.

23. Sanmartin-Gajardo I, Vianna JRS. Pollination of nematanthus brasiliensis: an epiphytic gesneriaceae endemic to the southeastern Atlantic forests of brazil. Selbyana. 2010;30:216-20.

24. Vasconcelos M, Lombardi J. Hummingbirds and their flowers in the campos rupestres of southern Espinhaco range. Brazil Melopsittacus. 2001;4:3-30.

25. Wolowski M, Ashman T, Freitas L. Community-wide assessment of pollen limitation in hummingbird-pollinated plants of a tropical montane rain forest. Ann Bot. 2013:112:903-10.

26. Chautems A. Révision taxonomique et possibilités d'hybridisations de Nematanthus Schrader (Gesneriaceae), genre endémique de la forêt côtière brésilienne. Dissertationes Botanicae. 1988;112:1-226.

27. Perret M, Chautems A, De Araujo AO, Salamin N. Temporal and spatia origin of Gesneriaceae in the New World inferred from plastid DNA sequences. Bot J Linn Soc. 2013:171:61-79.

28. Drummond AJ, Suchard MA, Xie D, Rambaut A. Bayesian Phylogenetics with BEAUti and the BEAST 1.7. Mol Biol Evol. 2012:29:1969-73.

29. Ronquist F, Teslenko M, van der Mark P, Ayres DL, Darling A, Hohna S, et al. MrBayes 3.2: efficient Bayesian phylogenetic inference and model choice across a large model space. Syst Biol. 2012;61:539-42.

30. Paradis E, Claude J, Strimmer K. APE: analyses of phylogenetics and evolution in R language. Bioinformatics. 2004;20:289-90.

31. Beaulieu JM, O'Meara BC, Donoghue MJ. Identifying hidden rate changes in the evolution of a binary morphological character: the evolution of plant habit in campanulid angiosperms. Syst Biol. 2013;62:725-37.

32. Dray $S$, Dufour AB. The ade4 package: implementing the duality diagram for ecologists. J Stat Softw. 2007;22:1-20.

33. Hijmans RJ, Cameron SE, Parra JL, Jones PG, Jarvis A. Very high resolution interpolated climate surfaces for global land areas. Int J Climatol. 2005;25:1965-78
34. Cooper N, Jetz W, Freckleton RP. Phylogenetic comparative approaches for studying niche conservatism. J Evol Biol. 2010;23:2529-39.

35. Doledec S, Chessel D, Gimaret-Carpentier C. Niche separation in community analysis: a new method. Ecology. 2000;81:2914-27.

36. Harmon LJ, Weir JT, Harmon Luke CD, Glor RE, Challenger W. GEIGER: investigating evolutionary radiations. Bioinformatics. 2008;24:129-31.

37. Blomberg SP, Garland T, Ives AR. Testing for phylogenetic signal in comparative data: behavioral traits are more labile. Evolution. 2003;57:717-45.

38. Revell $\amalg$. phytools: an $R$ package for phylogenetic comparative biology (and other things). Methods Ecol Evol. 2012;3:217-23.

39. Revell $\sqcup$. Two new graphical methods for mapping trait evolution on phylogenies. Methods Ecol Evol. 2013;4:754-9.

40. Ackerly D. Conservatism and diversification of plant functional traits Evolutionary rates versus phylogenetic signal. Proc Natl Acad Sci U S A. 2009;106:19699-706.

41. Hansen TF. Stabilizing selection and the comparative analysis of adaptation. Evolution. 1997;51:1341-51.

42. Corlay, S. Properties of the Ornstein-Uhlenbeck bridge. 2014; ArXiv:1310.5617v4

43. Brown $\mathrm{JH}$, Kodric-brown A. Convergence, competition, and mimicry in a temperate community of hummingbird-pollinated flowers. Ecology. 1979;60:1022-35

44. Clark JL, Zimmer EA. A preliminary phylogeny of Alloplectus (Gesneriaceae): Implications for the evolution of floral resupination. Syst Biol. 2003;28:365-75.

45. Clark JL. Systematics of Glossoloma (Gesneriaceae). Syst Bot Monogr. 2009:88:1-128

46. Fenster CB, Armbruster WS, Wilson P, Dudash MR, Thomson JD. Pollination syndromes and floral specialization. Annu Rev Ecol Evol Syst. 2004:35:375-403.

47. Castellanos MC, Wilson P, Thomson JD. "Anti-bee" and "pro-bird" changes during the evolution of hummingbird pollination in Penstemon flowers. J Evol Biol. 2004;17:876-85

48. Clark JL, Clavijo L, Muchhala N. Convergence of anti-bee pollination mechanisms in the Neotropical plant genus Drymonia (Gesneriaceae). Evol Ecol. 2015:29:355-77.

49. Muchhala N, Potts M. Character displacement among bat-pollinated flowers of the genus Burmeistera: analysis of mechanism, process and pattern. Proc R Soc B. 2007:274:2731-7.

50. Armbruster WS, Muchhala N. Associations between floral specialization and species diversity: cause, effect, or correlation ? Evol Ecol. 2009;23:159-79.

51. Muchhala N, Johnsen S, Smith SD. Pollniation shapes flower color variation in Andean Solanaceae. Evolution. 2014:68(8):2275-86.

52. Jakob SS, Heibl C, Rödder D, Blattner FR. Population demography influences climatic niche evolution: evidence from diploid American Hordeum species (Poaceae). Mol Ecol. 2010;19:1423-38.

53. Litsios G, Wüest RO, Kostikova A, Forest F, Lexer C, Linder HP, et al. Effects of a fire response trait on diversification in replicated radiations. Evolution. 2013;68:453-65.

54. Kostikova A, Litsios G, Salamin N, Pearman PB. Linking life-history traits, ecology, and niche breadth evolution in North American eriogonoids (Polygonaceae). Am Nat. 2013;182:760-74.

55. Oliveira-Filho AT, Fontes MAL. Patterns of floristic differentiation among Atlantic forests in southeastern Brazil and the influence of climate. Biotropica. 2000;32:793-810

56. Webb TJ, Woodward Fl, Hannah L, Gaston KJ. Forest cover-rainfall relationships in a biodiversity hotspot : the Atlantic forest of Brazil. Ecol Appl. 2005:15:1968-83.

57. Perret M, Chautems A, Spichiger R. Dispersal-vicariance analyses in the Tribe Sinningieae (Gesneriaceae): A clue to understanding biogeographical history of the Brazilian Atlantic forest. Ann Missouri Bot Gard. 2006;93:340-58.

58. Perret M, Chautems A, Spichiger R, Barraclough TG, Savolainen V. The geographical pattern of speciation and floral diversification in the neotropics: the tribe Sinningieae (Gesneriaceae) as a case study. Evolution. 2007;61:1641-60 